\title{
NUCLEAR SPIN POLARIZATION IN SILICON NANOSTRUCTURES WITH CHARGE CARRIER INJECTION
}

\author{
A. L. Danilyuk ${ }^{*}$ and V. E. Borisenko
}

UDC 539.143.5

We theoretically examine injection polarization of nuclear spins in silicon nanostructures with hyperfine interaction of nuclei with excited triplet states. We predict the possibility of the appearance of self-sustaining nuclear spin polarization, initiated by an external field. We show that if the external magnetic field is varied, we observe up to a 600-fold jump in the number of spin-polarized nuclei. A similar up to 40-fold jump also appears as the charge carrier injection rate increases.

Key words: nuclear polarization, silicon, hyperfine interaction, triplet state, nanostructure.

Introduction. Study of the behavior of electronic and nuclear spins in nanostructures upon excitation of their electronic subsystem is important for spintronics and quantum calculations. Due to the insufficient sensitivity of traditional rf spectroscopy methods, optical and electrical polarization and detection methods are used for these purposes [1-3]. Using electrical methods for metrology and control of spin ensembles leads to full-fledged integration of spin systems into semiconductor electronics [4]. From this standpoint, nuclear polarization (controllable by excitation of the electronic subsystem) is promising. For example, in silicon it is due to hyperfine interaction of the ${ }^{29} \mathrm{Si}$ nuclei with donor or triplet centers [5-7]. Substantial polarization of the nuclei in the first case is achieved by excitation of the electronic subsystem with circularly polarized light in compensated silicon, containing donor and acceptor centers; in the second case, by excitation with unpolarized light in a magnetic field. In this case, the degree of nuclear polarization reaches $5 \%-8 \%$ of the concentration of silicon nuclei as a result of hyperfine interaction with excited triplet centers of the $\mathrm{Si}-\mathrm{S} 1$ type [7]. This effect is promising for development of electronic control of nuclear polarization in silicon nanostructures.

The aim of this work was to model injection polarization of silicon nuclear spins in silicon nanostructures when they interact with triplet states, taking into account the magnetic field created by the nuclei.

The model. Let us describe the contact interaction of the nuclei with a triplet center with spin $S=1$ using the familiar Hamiltonian in [7, 8] which, taking into account the field $\mathbf{H}_{\mathrm{N}}=h_{\mathrm{N}}\langle\mathbf{I}\rangle$ created by the nuclei at the electron, has the form:

$$
H=g_{S} \mu_{\mathrm{B}}\left[\mathbf{H}_{0}+h_{\mathrm{N}}\langle\mathbf{I}\rangle\right] \mathbf{S}+D\left[S_{z}^{2}-(1 / 3) \mathbf{S}^{2}\right]+E\left(S_{x}^{2}-S_{y}^{2}\right)+\hbar A \mathbf{S I},
$$

where $\mu_{\mathrm{B}}$ is the Bohr magneton; $g_{S}$ is the $g$ factor for the electron of the triplet center; $\mathbf{S}$ is the electronic spin; $\mathbf{I}$, $\langle\mathbf{I}\rangle$ are the nuclear spin and its average value; $S_{x}, S_{y}, S_{z}$ are the spatial components of the electron spin; $A$ is the electron-nuclear spin-spin coupling constant; $\mathbf{H}_{0}$ is the external magnetic field; $D, E$ are the fine structure parameters of the center; $h_{\mathrm{N}}$ is the maximum nuclear field at the electron.

If the magnetic field is not parallel to any of the axes of the triplet center, then the spin eigenfunctions of the triplet state in the form of linear combinations look like [9]:

\footnotetext{
*To whom correspondence should be addressed.
}

Belarussian State University of Information Science and Electronics, 6 ul. P. Brovki, Minsk, 220013, Belarus. E-mail: danilyuk@nano-center.org. Translated from Zhurnal Prikladnoi Spektroskopii, Vol. 73, No. 5, pp. 647-653, September-October, 2006. Original article submitted April 4, 2006. 


$$
\psi_{k}=\sum_{j} c_{j}^{k} T_{j}
$$

Here $T_{X}, T_{Y}, T_{Z}$ are the basis functions in zero magnetic field; $c_{j}^{k}$ are coefficients; $k=1,2,3 ; j=X, Y, Z$ are the axes of the triplet center: $X=(1 / 3) D-E, Y=(1 / 3) D+E, Z=-(1 / 3) D$.

Taking into account (2), according to [8] the secular equation for the Hamiltonian (1) in the considered case has the form:

$$
\left|\begin{array}{ccc}
X-W & -i g \mu_{\mathrm{B}}\left(H_{z}+h_{\mathrm{N}}\left\langle I_{z}\right\rangle\right) & i g \mu_{\mathrm{B}}\left(H_{y}+h_{\mathrm{N}}\left\langle I_{y}\right\rangle\right) \\
i g \mu_{\mathrm{B}}\left(H_{z}+h_{\mathrm{N}}\left\langle I_{z}\right\rangle\right) & Y-W & i g \mu_{\mathrm{B}}\left(H_{x}+h_{\mathrm{N}}\left\langle I_{x}\right\rangle\right) \\
-i g \mu_{\mathrm{B}}\left(H_{y}+h_{\mathrm{N}}\left\langle I_{y}\right\rangle\right) & i g \mu_{\mathrm{B}}\left(H_{x}+h_{\mathrm{N}}\left\langle I_{x}\right\rangle\right) & Z-W
\end{array}\right|=0,
$$

where $H_{x}, H_{y}, H_{z}$ are the spatial components of the external magnetic field; $\left\langle I_{x}\right\rangle,\left\langle I_{y}\right\rangle,\left\langle I_{z}\right\rangle$ are the spatial components of the average nuclear spin.

From (3), we obtain a cubic equation of the form

$$
W^{3}+p W+q=0
$$

in which

$$
\begin{gathered}
p=-\left[\left(g \mu_{\mathrm{B}}\right)^{2}\left(H_{x}+h_{\mathrm{N}}\left\langle I_{x}\right\rangle\right)^{2}+\left(g \mu_{\mathrm{B}}\right)^{2}\left(H_{y}+h_{\mathrm{N}}\left\langle I_{y}\right\rangle\right)^{2}+\left(g \mu_{\mathrm{B}}\right)^{2}\left(H_{z}+h_{\mathrm{N}}\left\langle I_{z}\right\rangle\right)^{2}+(1 / 3) D^{2}+E^{2}\right]<0, \\
q=(D / 3)\left[\left(g \mu_{\mathrm{B}}\right)^{2}\left(H_{x}+h_{\mathrm{N}}\left\langle I_{x}\right\rangle\right)^{2}+\left(g \mu_{\mathrm{B}}\right)^{2}\left(H_{y}+h_{\mathrm{N}}\left\langle I_{y}\right\rangle\right)^{2}-2\left(g \mu_{\mathrm{B}}\right)^{2}\left(H_{z}+h_{\mathrm{N}}\left\langle I_{z}\right\rangle\right)^{2}+(2 / 9) D^{2}-2 E^{2}\right] .
\end{gathered}
$$

For the condition $Q=(p / 3)^{3}+(q / 2)^{2}<0$, a trigonometric solution of Eq. (4) is valid which, according to [10], has the form:

$$
W_{1}=2 \sqrt{-p / 3} \cos (\alpha / 3), \quad W_{2,3}=-2 \sqrt{-p / 3} \cos [(\alpha / 3) \pm(\pi / 3)],
$$

where $\cos \alpha=-q / \sqrt{-(p / 3)^{3}}$.

After transformations, we obtain

$$
\cos \alpha=-\frac{\sqrt{3}}{2} \frac{\left[a_{x}^{2}+a_{y}^{2}-2 a_{z}^{2}+(2 / 9)-\left(2 E^{2} / D^{2}\right)\right]}{\left[a_{x}^{2}+a_{y}^{2}+a_{z}^{2}+(1 / 3)+\left(E^{2} / D^{2}\right)\right]^{3 / 2}},
$$

where $a_{x, y, z}=g \mu_{\mathrm{B}}\left(H_{x, y, z}+h_{\mathrm{N}}\left\langle I_{x, y, z}\right\rangle\right) / D$ are the normalized spatial components of the magnetic field $\mathbf{H}_{0}+h_{\mathrm{N}}\langle\mathbf{I}\rangle$.

Note that for $Q>0$ and $p<0$, Eq. (4) has one real root and two complex conjugate roots.

Starting from Hamiltonian (1), in [11] expressions were obtained for the coefficients of the wave functions in (2), valid for $h_{\mathrm{N}}\langle\mathbf{I}\rangle=0$. Taking into account the nuclear field:

$$
\begin{aligned}
& \left|c_{x}^{k}\right|^{2}=\left[a_{y}^{2} Y_{k}^{2}+a_{z}^{2} a_{x}^{2}\right] V\left(X_{k}, Y_{k}\right)^{-1}, \\
& \left|c_{y}^{k}\right|^{2}=\left[a_{x}^{2} X_{k}^{2}+a_{z}^{2} a_{y}^{2}\right] V\left(X_{k}, Y_{k}\right)^{-1}, \\
& \left|c_{z}^{k}\right|^{2}=\left[a_{z}^{2}-X_{k} Y_{k}\right]^{2} V\left(X_{k}, Y_{k}\right)^{-1},
\end{aligned}
$$

where $Y_{k}=(Y / D)-\left(W_{k} / D\right), X_{k}=(X / D)-\left(W_{k} / D\right), V\left(X_{k}, Y_{k}\right)=a_{y}^{2} Y_{k}^{2}+a_{x}^{3} X_{k}^{2}+a_{z}^{2}\left(a_{x}^{2}+a_{y}^{2}\right)+\left[X_{k}^{2} Y_{k}^{2}-a_{z}^{2}\right]^{2}$. 
Expressions (7), (8), and (9)-(11) allow us to calculate the energies of the triplet states and the squares of the coefficients for the wave functions (2) based on the trigonometric solution of the secular equation we have proposed, taking into account the nuclear field in the case when the external field is not parallel to any of the axes of the triplet center.

For a magnetic field parallel to some axis of the triplet center, the expressions for the energies of the triplet states are simplified. If the field is parallel to the $z$ axis $(H \| O z)$, then according to [8, 11], the energies of the states for the considered case have the form:

$$
W_{1,3}=(D / 3) \pm E \sqrt{1+a_{E}^{2}}, \quad W_{2}=-2 D / 3
$$

where taking into account the nuclear field, we have the coefficient $a_{E}=g \mu_{\mathrm{B}}\left(H_{z}+h_{\mathrm{N}}\left\langle I_{z}\right\rangle / E\right.$.

If the field is parallel to the $x$ axis $(H \| O x)$ :

$$
W_{1,2}=-\frac{D-3 E}{6} \pm \frac{(D+E)}{2} \sqrt{1+a_{D x}^{2}}, \quad W_{3}=(D / 3)-E
$$

where $a_{D x}=2 g \mu_{\mathrm{B}}\left(H_{x}+h_{\mathrm{N}}\left\langle I_{x}\right\rangle\right) /(D+E)$.

If the field is parallel to the $y$ axis $\left(H \| O_{y}\right)$ :

$$
W_{1}=(D / 3)+E, \quad W_{2,3}=-\frac{D+3 E}{6} \mp \frac{(D-E)}{2} \sqrt{1+a_{D y}^{2}},
$$

where $a_{D y}=2 g \mu_{\mathrm{B}}\left(H_{y}+h_{\mathrm{N}}\left\langle I_{y}\right\rangle\right) /(D-E)$.

Based on (12)-(14), we obtain

$$
\left|c_{1}\right|^{2}=\frac{\left(1+\sqrt{1+a_{p}^{2}}\right)^{2}}{\left(1+\sqrt{1+a_{p}^{2}}\right)^{2}+a_{p}^{2}}, \quad\left|c_{2}\right|^{2}=\frac{\left(1-\sqrt{1+a_{p}^{2}}\right)^{2}}{\left(1-\sqrt{1+a_{p}^{2}}\right)^{2}+a_{p}^{2}}
$$

where for $H \| O z c_{1}=c_{y}^{1}=c_{x}^{3}, c_{2}=c_{x}^{1}=c_{y}^{3}, a_{p}=a_{E}$; for $H \| O x c_{1}=c_{y}^{1}=c_{z}^{2}, c_{2}=c_{y}^{2}=c_{z}^{1} a_{p}=a_{D x}$; for $H \| O y$ $c_{1}=c_{x}^{3}=c_{z}^{1}, c_{2}=c_{z}^{2}=c_{y}^{3}, a_{p}=a_{D y}$.

If the field makes an angle with the $z$ axis that is not very large, $\theta \leq \pi / 6$, then the energies of the triplet states and the squares of the wave functions have the form [7]:

$$
\begin{gathered}
W_{1}=(D / 3)+h_{z}, \quad W_{2,3}=-(1 / 2)\left[(D / 3)+h_{z}\right] \pm \sqrt{\left(h_{z}-D\right)^{2}-2 h_{z}^{2} \sin ^{2} \theta}, \\
\left|c_{1,2}\right|^{2}=\left|c_{4,3}\right|^{2}=(1 / 2) \pm(1 / 2)\left(h_{z}-D\right) / \sqrt{\left(h_{z}-D\right)^{2}-2 h_{z}^{2} \sin ^{2} \theta},
\end{gathered}
$$

where $h_{z}=g_{S} \mu_{\mathrm{B}}\left(H_{z}+h_{\mathrm{N}}\left\langle I_{z}\right\rangle\right)$.

Nuclear polarization arises as a result of hyperfine interaction of the nuclear spins with the excited triplet states, which are in a nonequilibrium spin state [6, 7]. Let us consider an isotropic hyperfine interaction, the Hamiltonian of which has the form [7, 12]:

$$
H_{H F I}=A \hbar\left[S_{z} I_{z}+(1 / 2)\left(S_{+} I_{-}+S_{-} I_{+}\right)\right]
$$

where the terms $S_{+} I_{-}$and $S_{-} I_{+}$correspond to transitions with simultaneous changes in the projections of the electronic and nuclear spins $\Delta m_{S}= \pm 1$ and $\Delta m_{I}=-( \pm 1)$. Such transitions occur as a result of modulation of the hyperfine interaction.

By introducing the probabilities of the relaxation transitions $w_{i j}$ changing the projection of the nuclear spin $m_{I}$, which are accompanied by transitions of electrons between the states $T_{j}$, in analogy with [13] let us construct the ki- 
netic equations for the populations of the nuclear and electronic magnetic sublevels, leading to the following equation for the degree of nuclear polarization in the general case:

$$
P_{n}-P_{n 0}=\frac{(3 / 2) f\left[\left(P_{1}-P_{10}\right)\left(w_{12}-w_{21}+w_{13}-w_{31}\right)+\left(P_{2}-P_{20}\right)\left(w_{13}-w_{31}+w_{23}-w_{32}\right)\right]}{w_{12}+w_{12}+w_{13}+w_{13}+w_{23}+w_{32}},
$$

where $P_{1}=\left(n^{+}-n^{0}\right) / n, P_{2}=\left(n^{0}-n^{-}\right) / n$ are the polarizations of the electrons; $n=n^{+}+n^{0}+n^{-}, n^{+}, n^{0}, n^{-}$are the populations of the corresponding levels of the triplet center; $f$ is the nuclear polarization leakage factor; $P_{n 0}$ is the equilibrium value of the nuclear polarization.

Let us determine the polarizations of the electrons, taking into account spin relaxation in the excited triplet state, using a system of kinetic equations that we write in analogy with the system presented in [14]:

$$
\begin{aligned}
& \frac{d n^{+}}{d t}=\Gamma^{+}-n^{+} R^{+}-\left(n^{+}-n^{0}\right) W_{(+\leftrightarrow 0)}-\left(n^{+}-n^{-}\right) W_{(+\leftrightarrow-1)}-\frac{n^{+}}{\tau}-\frac{1}{2 \tau_{S}}\left(2 n^{+}-n^{0}-n^{-}\right), \\
& \frac{d n^{0}}{d t}=\Gamma^{0}-n^{0} R^{0}-\left(n^{0}-n^{+}\right) W_{(+\leftrightarrow 0)}-\left(n^{0}-n^{-}\right) W_{(0 \leftrightarrow-1)}-\frac{n^{0}}{\tau}-\frac{1}{2 \tau_{S}}\left(2 n^{0}-n^{+}-n^{-}\right), \\
& \frac{d n^{-}}{d t}=\Gamma^{-}-n^{-} R^{-}-\left(n^{-}-n^{0}\right) W_{(0 \leftrightarrow-1)}-\left(n^{-}-n^{+}\right) W_{(+\leftrightarrow-1)}-\frac{n^{-}}{\tau}-\frac{1}{2 \tau_{S}}\left(2 n^{-}-n^{+}-n^{0}\right),
\end{aligned}
$$

where $\Gamma^{+}, \Gamma^{0}, \Gamma^{-}$are the rates at which the triplet levels $|+1\rangle,|0\rangle,|-1\rangle$ are populated; $W_{(+\leftrightarrow))}, W_{(0 \leftrightarrow-1)}, W_{(+\leftrightarrow-1)}$ are the corresponding probabilities of the transitions between the states $T_{+}, T_{0}, T_{-} ; R^{+}, R^{0}, R^{-}$are the probabilities of transition from the triplet states of the center to the singlet ground state due to the presence of spin-orbit coupling; $\tau$ is the lifetime of the triplet state; $\tau_{S}$ is its spin relaxation time.

From (19), taking into account (20) and the fact that the probabilities of the relaxation transitions $w_{i j}$ are proportional to the squares of the corresponding mixing coefficients of the wave function (2) [7], we obtain an expression for the degree of nuclear polarization. In the general case, the analytical expression for the degree of polarization is unwieldy, and numerical solution of the system of equations (7)-(11), (19), (20) is optimal.

If the external magnetic field and the nuclear field are parallel to the an axis of the triplet center, then the description is simplified. If these fields are parallel to the $z$ axis or make a not very large angle $\theta$ with it, then the electronic polarizations in the presence of injection of nonequilibrium charge carriers are expressed in the form [7]:

$$
P_{1}-P_{10}=\tau_{S} \frac{\left|c_{1}\right|^{2} \Gamma-P_{10}}{\tau+\tau_{S}}, \quad P_{2}-P_{20}=\tau_{S} \frac{\left|c_{1}\right|^{2} \Gamma-\left|c_{2}\right|^{2} \Gamma-P_{20}}{\tau+\tau_{S}},
$$

where $\Gamma=\left(\Gamma^{+}-\Gamma^{0}\right) /\left(\Gamma^{+}+\Gamma^{0}+\Gamma^{-}\right)$, the $P_{10}, P_{20}$ are the equilibrium values of the electronic polarizations of the triplet states.

The degree of nuclear polarization in this case, neglecting the nuclear field [7], is:

$$
P_{n}-P_{n 0}=\frac{3}{2} f \frac{\tau_{S}}{\tau_{S}+\tau} \frac{2 \Gamma\left|c_{1}\right|^{2}\left|c_{2}\right|^{2}-P_{10}-P_{20}\left|c_{1}\right|^{2}}{1+\left|c_{1}\right|^{4}+\left|c_{2}\right|^{4}}
$$

Taking into account the nuclear field at the triplet center and assuming that $\left\langle I_{x, y, z}\right\rangle=P_{n x, y, z} / 2$, we obtain an expression for the degree of nuclear polarization in the case when the field makes a not very large angle $\theta$ with the $z$ axis, for the condition that $P_{n}>>P_{n 0}$ :

$$
P_{n z}=F\left(P_{n z}\right)=\left(\frac{b}{2}\right) \frac{\mu\left[\left(H_{z} / H_{D}\right)+a_{D} P_{n z}\right]^{2}}{3 \mu\left[\left(H_{z} / H_{D}\right)+a_{D} P_{n z}\right]^{2}+4\left[\left(H_{z} / H_{D}\right)+a_{D} P_{n z}-1\right]^{2}},
$$




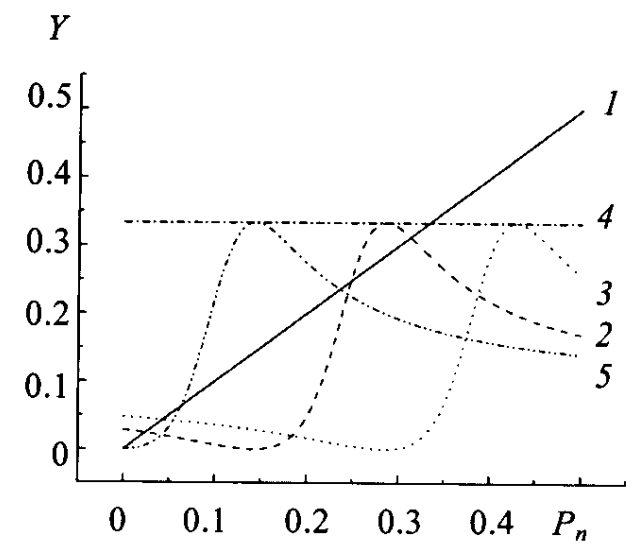

Fig. 1 Graphical solution of Eq. (23) for $b=2, \theta=\pi / 6, a_{D}=7: Y=P_{n}(1)$, $F\left(P_{n z}\right)(2,3,5), P_{n H}(4) ; H_{z} / H_{D}=0(5),-1(2)$, and -2 (3).

where $b=3 f\left[\tau_{S} /\left(\tau+\tau_{S}\right)\right] \Gamma_{S} ; \mu=2 \sin ^{2} \theta ; a_{D}=h_{\mathrm{N}} / 2 H_{D} ; H_{D}=\left(g \mu_{\mathrm{B}}\right)^{-1} D$.

For the case when the external field and the nuclear field are parallel to the $z$ axis:

$$
P_{z}=2 b\left[H_{z} / H_{E}+\left(h_{\mathrm{N}} / 2 H_{E}\right) P_{n z}\right]^{2}\left(4+3\left[H_{z} / H_{E}+\left(h_{\mathrm{N}} / 2 H_{E}\right) P_{n z}\right]^{2}\right)^{-1},
$$

where $H_{E}=\left(g \mu_{\mathrm{B}}\right)^{-1} E$.

Analogously, for the cases when these fields are parallel to the $x$ and $y$ axes:

$$
\left.\left.P_{n x, y}=2 b\left[H_{x, y} /\left(H_{D} \pm H_{E}\right)\right] P_{n x, y}\right]^{2}\left(4+3\left[H_{x, y} /\left(H_{D} \pm H_{E}\right)\right] P_{n x, y}\right]^{2}\right)^{-1} .
$$

Eqs. (23)-(25) determine the steady-state values of the degree of nuclear polarization for hyperfine interaction of the nuclei with the triplet centers in an external field, taking into account the nuclear field for cases when the field is parallel to an axis of the center or makes a not very large angle with the $z$ axis. The equations obtained are valid for silicon nanostructures containing excited triplet centers with spin $S=1$. These centers effectively arise at dangling bonds of silicon atoms in a $\mathrm{Si} / \mathrm{SiO}_{2}$ nanostructure or in silicon nanocrystallites of size 2-3 nm in the presence of injection of nonequilibrium charge carriers, the rate of which determines the lifetime of the triplet state and the rates at which its levels are populated.

Calculation results and discussion. For the case when the direction of the external magnetic field and the nuclear field make an angle $\theta$ with the $z$ axis of the triplet center, we obtain the following results. In the absence of a nuclear field at the triplet centers, the external magnetic field $H_{z}$ creates the maximum degree of nuclear polarization, determined by the single value $P_{n}=P_{n H}$ for $H_{z}=H_{D}$ (Fig. 1, curve 4). In the presence of a nuclear field and in the absence of an external magnetic field, a zero solution additionally arises. If the condition $a_{D}=h_{\mathrm{N}} / 2 H_{D}>a_{\mathrm{cr}}^{D}$ or $b>$ $b_{\text {cr }}$ is satisfied, then two more positive solutions arise for Eq. (23) (Fig. 1, curve 5). Thus the parameters $a_{D}$ and $b$ are critical; when they exceed certain values, new solutions arise for the polarization equation (23). The curve showing the degree of nuclear polarization in the steady state vs. the parameter $a_{D}$ or $b$ has a shape typical of a second-order phase transition (Fig. 2). The largest of the indicated solutions corresponds to $P_{n H}$ for the phase transition parameters, but as the field $h_{\mathrm{N}}$ increases, all other conditions being equal, its value drops. Application of an external magnetic field in the same direction as the nuclear field leads to loss of the zero and intermediate solutions. Only the largest solution remains, which (as shown by the analysis) is stable. In this case, the degree of nuclear polarization decreases. An external magnetic field in the opposite direction relative to the nuclear field leads to an increase in all three of the indicated solutions (Fig. 1, curve 2). However, this is observed only up to a certain value of the external field, exceeding which in absolute value leads to a loss of the largest and intermediate solutions (curve 3). We interpret the identified effect as the appearance of self-sustaining nuclear polarization as a result of the intrinsic nuclear field, initiated and modulated by the external magnetic field. 


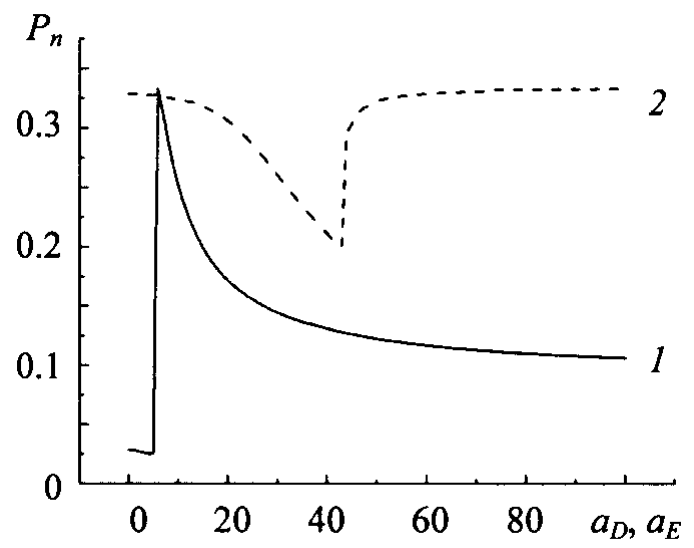

Fig. 2 Degree of polarization of ${ }^{29} \mathrm{Si}$ nuclei in the stable state as a function of the critical parameters $a_{D}$ and $a_{E}$ for $b=2: 1$ ) field direction makes $\pi / 6$ angle with the $z$ axis, $H_{z} / H_{D}=-1$; 2) field is parallel to the $z$ axis, $H_{z} / H_{E}=-10$.

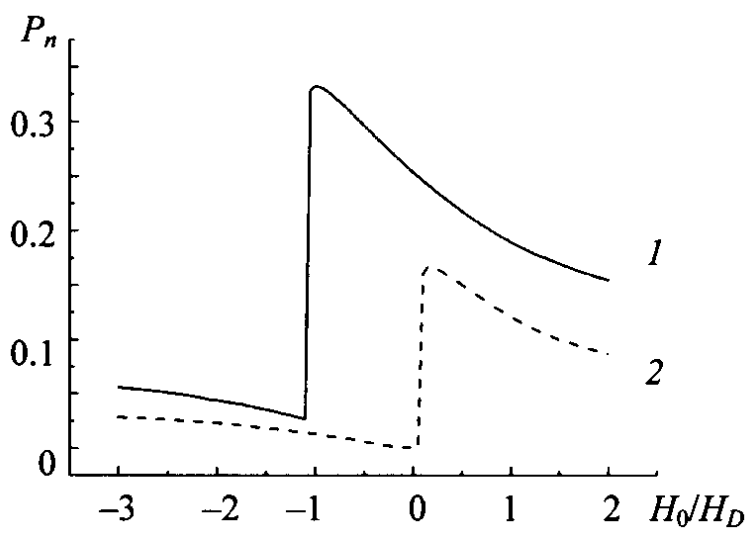

Fig. 3 Jump in nuclear polarization in an external magnetic field: 1) $a_{D}=6$, $b=2$;2) $a_{D}=5, b=1$.

Initiation means that this effect arises only in the presence of an initial nuclear polarization, while modulation means that subsequent variation of the external magnetic field leads to a jump in the degree of nuclear polarization (Fig. 3). An increase in the external magnetic field in the same direction as the nuclear field is accompanied by a jump in the degree of nuclear polarization by no more than a factor of $2-3$. In an external magnetic field in the opposite direction, the jump in the degree of polarization is as high as by a factor of 10-13 for $b=2, a_{D}=6$ and increases up to 600 -fold for $b=1, a_{D}=5$ (Fig. 3). The difference between the absolute value of the degree of polarization is less in the second case than in the first case.

The electric field in this case is controlled by modulation of the parameter $b$. This modulation occurs as the injection rate is varied, which leads to a change in the ratio between the lifetime $\tau$ and the spin relaxation time of the triple center $\tau_{S}$. For example, as the injection rate increases, the recombination rate increases, which decreases the lifetime of the excited triplet state and increases the value of $b$. As the parameter $b$ varies, a jump in the polarization also appears (Fig. 4). The ratio achieved between the values of the polarization in this case is $20-40$ for $b=2$.

The characteristic time for such jumps corresponds to the polarization time for nearby nuclei, which we estimate on the basis of the model we developed for polarization of nuclei in a silicon/dielectric nanostructure [15]. For silicon nuclei ${ }^{29} \mathrm{Si}$, for their natural concentration this time is on the order of $10^{-4}$ to $10^{-3} \mathrm{sec}$, provided that spin diffusion is blocked. As the concentration of the ${ }^{29} \mathrm{Si}$ isotope increases, it decreases down to $10^{-6}$ to $10^{-5} \mathrm{sec}$. 


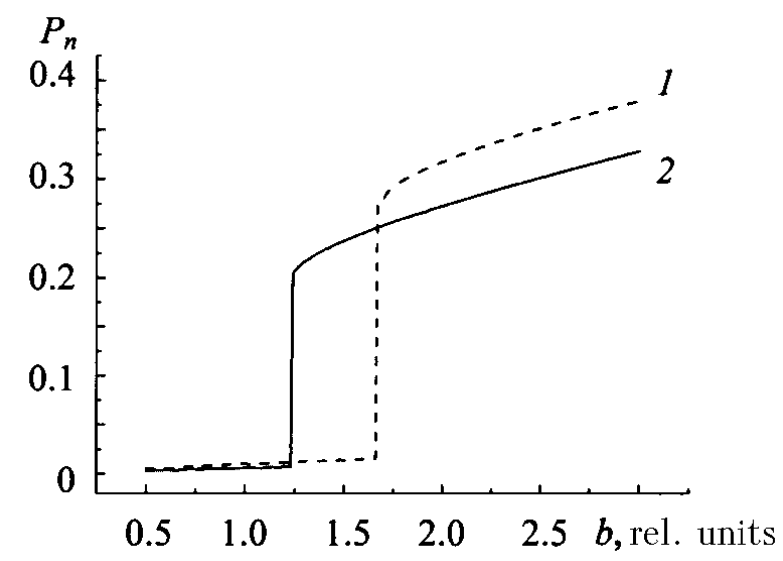

Fig. 4 Jump in nuclear polarization as the charge carrier injection rate increases: 1) $\left.a_{D}=6 ; H_{z} / H_{D}=-0.75 ; 2\right) a_{D}=7 ; H_{z} / H_{D}=-0.50$.

Note that for application of the identified effects, we should eliminate channels for leakage of nuclear polarization and maintain the necessary ratio between the times $\tau$ and $\tau_{S}$, which is possible when using silicon nanostructures.

Analogous results were obtained for the case when the nuclear field is parallel to an axis of the triplet center (Fig. 2, curve 2). In this case, we also observe phase transitions, which are controlled by the magnetic or electric field (potential), the jump is smaller and is no greater than by a factor of 1.5-2. The calculations performed using the equations for the general case have confirmed the results obtained in the situation when the nuclear field makes an angle $\theta$ with the $z$ axis of the triplet center.

The mechanism for the appearance of self-sustaining polarization is as follows. Injection of nonequilibrium charge carriers in nanostructures is responsible for the transition of the triplet centers to the excited state. In an external magnetic field, the triplet center acquires the spin polarization needed for initial polarization of the ${ }^{29} \mathrm{Si}$ nuclei. Over the course of $10^{-5}$ to $10^{-3} \mathrm{sec}$, polarization occurs for the nuclei surrounding the triplet center, i.e., nearby nuclei [15]. At the same time, relaxation processes begin for the nuclei at the triplet and other centers, and also spin transfer to more distant nuclei as a result of spin diffusion between nuclei. In this case, the spin diffusion process can be blocked as a result of a diffusion barrier [16], and also when using structures containing silicon nanocrystallites with passivated boundaries. After the external field is turned off, the triplet center is found in the magnetic field created by nearby nuclei. Such a field maintains the spin polarization of the triplet center, which in turn is responsible for nuclear polarization. Under the conditions considered above, this process is self-organized in such a way that the steady-state values of the nuclear and electronic polarizations are held constant. Self-sustaining polarization exists provided that the rate of spin transfer to distant nuclei, and also the rate of nuclear relaxation at other paramagnetic centers, which do not promote nuclear polarization, are substantially slower than the rate of spin exchange between the triplet center and nearby nuclei. The indicated effect arises jumpwise as the system parameters vary. It is analogous to a second-order phase transition. In this case, variation of the external magnetic field or the rate of charge carrier injection leads to a change in the phase transition conditions, which makes it possible to control the process of a jump in the degree of nuclear polarization.

In charge carrier injection as a result of an external electric potential, the process of polarization of the silicon nuclei can be controlled, such as by turning on and off the injection itself or by changing the type of injection (the ratio of electrons and holes) or the injection rate.

In $\mathrm{Si} / \mathrm{SiO}_{2}$ nanostructures, the triplet centers arise naturally at dangling silicon bonds $\mathrm{Si}-S 1$ [7]) at the interface. In a silicon nanostructure with another dielectric, they arise as a result of excitation of charge carriers in the silicon nanocrystallites [17]. In an external magnetic field in silicon nanocrystallites of size 2-3 nm, substantial polarization of nuclei for the magnetic isotope of silicon is possible using such states. In this case, nuclear polarization will be self-sustaining after removal of the magnetic field in the presence of excited charge carriers. Since the boundaries of the nanocrystallites are passivated, the wave function of the electron is localized in the nanocrystallites [18]. 
This makes it possible, by choosing the concentration of the magnetic isotopes and the size of the nanocrystallite, to control the nuclear polarization using an electric field, which is important for designing memory components based on nuclear spins and setting up quantum calculations.

The considered effect can be detected by using optical methods, such as from the change in the polarization of luminescence arising as a result of recombination processes at the triplet centers, or from decay of polarization of luminescence in a transverse magnetic field [19]. This effect can also be detected by an electric method, by measuring tunneling magnetoresistance in a silicon/dielectric nanostructure, which under the considered conditions depends substantially on the nuclear polarization.

Conclusion. Injection polarization of nuclear spins in silicon nanostructures with hyperfine interaction of silicon nuclei with excited triplet states leads to the appearance of self-sustaining nuclear polarization as a result of the intrinsic nuclear field. In this case, we observe a jump in the degree of nuclear polarization when the external magnetic field or the charge carrier injection rate is varied. The time for transition from one state to another depends on the concentration of magnetic isotopes of silicon, and varies from $10^{-3} \mathrm{sec}$ to $10^{-6} \mathrm{sec}$. When the magnetic field is varied, the change in the degree of nuclear polarization is as high as 600-fold. As the charge carrier injection rate increases, the increase in the degree of nuclear polarization at the jump is as high as by a factor of $20-40$. The considered effect can be used to design memory based on nuclear spins, controllable by electric potentials, and also in setting up quantum calculations.

\section{REFERENCES}

1. F. Meier and B. P. Zakharchenya, eds., Optical Orientation [Russian translation], Nauka, Leningrad (1989). [English original: Elsevier, Amsterdam (1984)].

2. J. M. Kikkawa and D. D. Awschalom, Science, 287, 473-476 (2000).

3. M. S. Brandt, S. E. Ready, and J. B. Boyce, Appl. Phys. Lett., 70, 188-190 (1997).

4. B. P. Zakharchenya and V. L. Korenev, Usp. Fiz. Nauk, 175, 629-635 (2005).

5. N. T. Bagraev and L. S. Vlasenko, Fiz. Tverd. Tela, 21, 120-133 (1979).

6. N. T. Bagraev and L. S. Vlasenko, Zh. Eksp. Teor. Fiz., 83, 2186-2200 (1982).

7. L. S. Vlasenko, Izv. Akad. Nauk SSSR, Ser. Fiz., 46, 469-475 (1982).

8. S. P. McGlynn, T. Azumi, and M. Kinoshita, Molecular Spectroscopy of the Triplet State [Russian translation], Mir, Moscow (1972). [English original: Prentice-Hall, Englewood Cliffs, New Jersey (1969).]

9. P. Kottis and R. Lefebvre, J. Chem. Phys., 41, 379-393 (1964).

10. G. A. Korn and T. M. Korn, Mathematical Handbook for Scientists and Engineers [Russian translation], Nauka, Moscow (1984). [English original: McGraw-Hill (1968).]

11. P. Kottis and R. Lefebvre, J. Chem. Phys., 39, 393-403 (1963).

12. A. Abragam, The Principles of Nuclear Magnetism [Russian translation], Inostr. Lit., Moscow (1963). [English original: Oxford (1961).]

13. N. T. Bagraev and L. S. Vlasenko, Zh. Eksp. Teor. Fiz., 75, 1743-1754 (1978).

14. L. S. Vlasenko, Izv. Akad. Nauk SSSR, Ser. Fiz., 47, 2325-2330 (1983).

15. A. L. Danilyuk and V. E. Borisenko, Zh. Prikl. Spektr., 72, 397-403 (2005).

16. G. R. Khutsishvili, Usp. Fiz. Nauk, 87, 211-254 (1965).

17. H. Heckler, D. Kovalev, G. Polisski, N. N. Zinov'ev, and F. Koch, Phys. Rev. B, 60, 7718-7721 (1999).

18. F. Bassani, L. Vervoot, I. Mihalescu, J. C. Vial, and F. A. d'Avitaya, J. Appl. Phys., 79, 4066-4071 (1996).

19. A. L. Danilyuk and V. E. Borisenko, Zh. Prikl. Spektr., 72, 524-529; 644-649 (2005). 